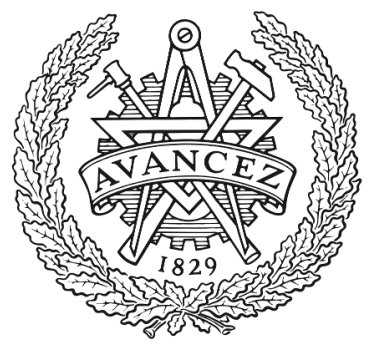

CHALMERS

UNIVERSITY OF TECHNOLOGY

\title{
Do Optomechanical Metasurfaces Run Out of Time?
}

Downloaded from: https://research.chalmers.se, 2023-04-26 07:21 UTC

Citation for the original published paper (version of record):

Viaene, S., Ginis, V., Danckaert, J. et al (2018). Do Optomechanical Metasurfaces Run Out of Time? Physical Review Letters, 120(19). http://dx.doi.org/10.1103/PhysRevLett.120.197402

N.B. When citing this work, cite the original published paper. 


\title{
Do Optomechanical Metasurfaces Run Out of Time?
}

\author{
Sophie Viaene, ${ }^{1,2}$ Vincent Ginis, ${ }^{1,3,}{ }^{*}$ Jan Danckaert, ${ }^{1}$ and Philippe Tassin ${ }^{2,1}$ \\ ${ }^{1}$ Applied Physics Research Group, Vrije Universiteit Brussel, Pleinlaan 2, B-1050 Brussel, Belgium \\ ${ }^{2}$ Department of Physics, Chalmers University of Technology, SE-412 96 Göteborg, Sweden \\ ${ }^{3}$ Harvard John A. Paulson School of Engineering and Applied Sciences, Harvard University, \\ 29 Oxford Street, Cambridge, Massachusetts 02138, USA
}

(Received 8 October 2017; published 11 May 2018)

\begin{abstract}
Artificially structured metasurfaces make use of specific configurations of subwavelength resonators to efficiently manipulate electromagnetic waves. Additionally, optomechanical metasurfaces have the desired property that their actual configuration may be tuned by adjusting the power of a pump beam, as resonators move to balance pump-induced electromagnetic forces with forces due to elastic filaments or substrates. Although the reconfiguration time of optomechanical metasurfaces crucially determines their performance, the transient dynamics of unit cells from one equilibrium state to another is not understood. Here, we make use of tools from nonlinear dynamics to analyze the transient dynamics of generic optomechanical metasurfaces based on a damped-resonator model with one configuration parameter. We show that the reconfiguration time of optomechanical metasurfaces is not only limited by the elastic properties of the unit cell but also by the nonlinear dependence of equilibrium states on the pump power. For example, when switching is enabled by hysteresis phenomena, the reconfiguration time is seen to increase by over an order of magnitude. To illustrate these results, we analyze the nonlinear dynamics of a bilayer cross-wire metasurface whose optical activity is tuned by an electromagnetic torque. Moreover, we provide a lower bound for the configuration time of generic optomechanical metasurfaces. This lower bound shows that optomechanical metasurfaces cannot be faster than state-of-the-art switches at reasonable powers, even at optical frequencies.
\end{abstract}

DOI: 10.1103/PhysRevLett.120.197402

The field of optomechanics [1,2] has generated several high-precision techniques to control and detect minute motions of a variety of objects by optimizing the interaction of light with mechanical degrees of freedom [3-5]. Conversely, optical forces and torques also provide a versatile mechanism to control electromagnetic responses. For example, optomechanical metasurfaces consist of a collection of subwavelength unit cells with optimized electromagnetic resonators that are connected by elastic elements which may move to oppose pump-induced forces [6-8]. The idea is to use the electromagnetic force of a pump beam to displace [6,9-12], deform $[13,14]$, or rotate $[9,15]$ electromagnetic resonators, so magnetic [6], chiral $[13,14,16]$, or other electromagnetic responses [9-11] may be tuned by the power of the pump. Optomechanical metasurfaces have been praised for their enhanced nonlinear response because hysteresis and jump phenomena allow processing light with light [17-20] and may extend the range of electromagnetic responses [21].

In this Letter, we demonstrate that hysteresis phenomena dramatically increase the reconfiguration time of optomechanical metasurfaces due to critical slowing-down, a phenomenon which is well established in other nonlinear systems [22-24]. Based on a damped-resonator model, we obtain a lower bound for the reconfiguration time which scales unfavorably with the frequency and power of the pump. As a result, the response times of optomechanical switches do not improve on the state of the art [20]. Figure 1(a) visualizes a generic two-dimensional optomechanical unit cell that consists of electromagnetic resonators connected to elastic components such as filaments, membranes, or substrates $[6,14]$. We assume that the configuration of the unit cell is described by one configuration parameter $\psi$, whose value changes when the unit cell is deformed from $\psi_{0}$ in the absence of a pump to a new equilibrium $\psi_{\mathrm{eq}}$ in the presence of a pump. Note that the electromagnetic action on the unit cell $P_{0} \mathcal{A}(\psi)$ is proportional to the power $P_{0}$ that is deposited on the unit cell, while its dependence on the configuration parameter $\mathcal{A}(\psi)$ results from resonances of the electromagnetic modes. At the equilibrium configuration, the electromagnetic action is balanced by a restoring action of the elastic elements $\mathcal{R}(\psi)$, which is to a very good approximation given by Hooke's law $\mathcal{R}(\psi)=-\kappa\left(\psi-\psi_{0}\right)$ [straight line in Fig. 1(b)]. Close to equilibrium, the net electromagnetic action on the unit cell results in an effective optical spring constant $\kappa_{\mathrm{opt}}=$ $\kappa-\left[\left(P_{0} \partial \mathcal{A}\left(\psi_{\mathrm{eq}}\right)\right) / \partial \psi\right][25]$ which determines if equilibrium states are stable $\left(\kappa_{\mathrm{opt}}>0\right)$ or unstable $\left(\kappa_{\mathrm{opt}}<0\right)$. For the sake of tunability, we assume that the pump beam is operated close to a resonance frequency $f_{0}$ of the 
(a) Optomechanical unit cell Electromagnetic action $\mathrm{A}(\psi)$ in equilibrium $\psi_{0}$

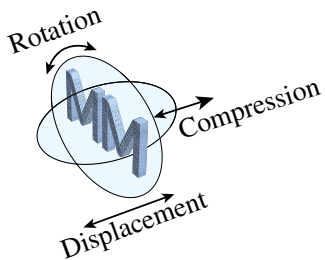

leads to a new equilibrium $\psi_{\text {eq }}$

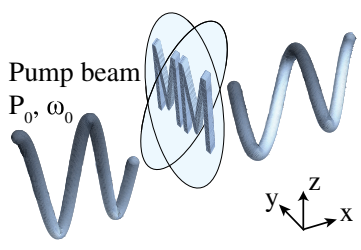

(b) Action $\mathrm{A}(\psi)$ for $\mathrm{P}_{0}=1$

(c) Equilibrium configuration $\psi_{\text {eq }}$

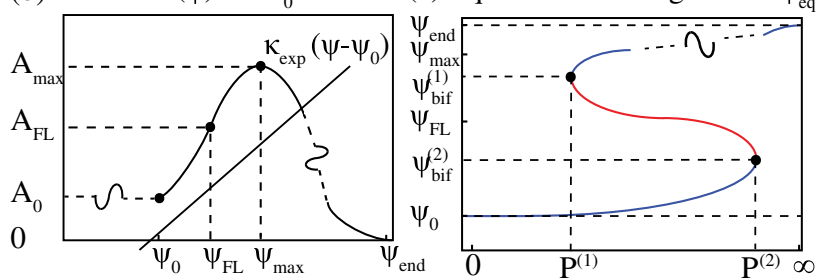

FIG. 1. Visualization of a generic optomechanical unit cell whose internal configuration $\psi$ changes to compensate for the electromagnetic action $P_{0} \mathcal{A}(\psi)$ on elastic elements with a restoring action $\kappa\left(\psi-\psi_{0}\right)$. (a) The initial equilibrium $\psi_{0}$ changes to $\psi_{\text {eq }}$ when a pump beam acts on the unit cell. (b) Equilibrium states correspond to intersections of the electromagnetic action of the pump and the restoring action of an elastic element with restoring parameter $\kappa$. (c) Equilibrium states are either stable (blue) or unstable (red) and may form hysteresis curves for specific values of $\psi_{0}$ due to the presence of an inflection point $\psi_{\mathrm{FL}}$.

metasurface, so the electromagnetic action $P_{0} \mathcal{A}(\psi)$ reaches a maximum at the value $\psi_{\max }$ of the reconfiguration parameter for which the metasurface is most resonant [Fig. 1(b)]. As explained in Fig. S1 of the Supplemental Material [26], the equilibrium states depend in a highly nonlinear way on the power of the pump and may form hysteresis curves [Fig. 1(c)]. An optomechanical metasurface with one configuration parameter will always generate a hysteresis curve for a particular range of parameter values $\psi_{0}$ [see Fig. S1(e) of the Supplemental Material [26] ] when the electromagnetic action has an inflection point $\psi_{\mathrm{FL}}$ [Fig. 1(b)]. This inflection point allows for multiple equilibrium states in between $\psi_{0}$ and $\psi_{\max }$, i.e., multiple intersections between the electromagnetic action and the restoring action in Fig. 1(b), and has to exist when the unit cell has multiple resonances, symmetries in terms of the configuration parameter, or when the electromagnetic action approaches zero, as in Refs. [7,15]. To investigate whether optomechanical metasurfaces may be used as a switch, we study the transient dynamics of unit cells as they evolve from one equilibrium configuration $\psi_{\mathrm{eq}}^{(1)}$ to another $\psi(2)$ under the influence of the time-averaged electromagnetic action. Note that the electromagnetic problem decouples from the mechanical problem because mechanical frequencies are much lower than the frequency of the pump beam. Therefore, we have extracted the electromagnetic action from finite-element simulations before solving for the nonlinear dynamics of the unit cell, for which the electromagnetic action is a nonideal source [22,27]. In particular, the unit cell is modeled as a damped resonator, whose equation of motion

$$
\mathcal{M} \frac{\partial^{2} \psi}{\partial t^{2}}+\gamma \frac{\partial \psi}{\partial t}+\kappa\left(\psi-\psi_{0}\right)=P_{0}\left\langle\mathcal{A}\left(\psi, f_{0}\right)\right\rangle
$$

contains the (moment of) inertia $\mathcal{M}$ of the movable parts of the unit cell, a damping term $\gamma$, a spring constant $\kappa$ due to an elastic element with equilibrium state $\psi_{0}$, and a time-averaged electromagnetic action $P_{0}\left\langle\mathcal{A}\left(\psi, f_{0}\right)\right\rangle$. We impose that the restoring parameter $\kappa$ is optimized, so the restoring action allows for a reasonable change $\Delta \psi$ of the configuration. In mathematical terms, at the desired power of operation $P_{0 \mathrm{op}}$, the restoring parameter is related to the maximal value of the electromagnetic action: $\kappa \Delta \psi=P_{0 \text { op }} \mathcal{A}_{\max }$. Importantly, $\kappa$ cannot be increased at will to improve the reconfiguration time. The equation of motion is solved in dimensionless coordinates with normalized parameters $\tilde{\gamma}=(\gamma / \mathcal{M}), \tilde{\kappa}=\left(\kappa / \mathcal{M} \tilde{\gamma}^{2}\right), \tilde{\mathcal{A}}=$ $\left(\mathcal{A} / \mathcal{M} \tilde{\gamma}^{2}\right)$ in terms of an evolution parameter $\tau=\tilde{\gamma} t$ on a two-dimensional phase plane $(\psi,(d \psi / d \tau))$,

$$
\frac{d \psi}{d \tau}=v, \frac{d v}{d \tau}=-v-\tilde{\kappa}\left(\psi-\psi_{0}\right)+P_{0}\left\langle\tilde{\mathcal{A}}\left(\psi ; f_{0}\right)\right\rangle .
$$

Throughout this Letter, we assume that the resonator is critically damped, i.e., $\tilde{\gamma}_{\text {crit }}=$ $2 \sqrt{(\kappa / \mathcal{M})-\left(P_{0} / \mathcal{M}\right)\left(\partial \mathcal{A}\left(\psi_{\text {eq }}\right) / \partial \psi\right)}$, so the unit cell converges in an optimal way. In the Supplemental Material [26] Figs. S10 and S11, the reconfiguration time of over- and underdamped resonators is shown to be higher. As a particular illustration of the time-averaged electromagnetic action $P_{0}\langle\tilde{\mathcal{A}}(\psi)\rangle$, we consider an optically active bilayer metasurface [28-30] consisting of cross wires that are connected by torsional wires as in Fig. 2(a). Other mechanisms to tune optically active metasurfaces can be found in Refs. [19,31-37]. The ability to turn a linearly polarized signal beam over an angle $\delta$ is determined by the relative orientation $\varphi$ of the crosses $[8,28,38]$, which provides the reconfiguration parameter of the metasurface. As explained in the Supplemental Material [26] and Refs. [39-41] therein, the electromagnetic torque and the optical activity $\delta$ have been extracted from full-wave numerical simulations for various angular orientations of the crosses and frequencies of the pump (see Figs. S3-S5 in the Supplemental Material [26]). The simulations were performed at $1 \mathrm{~W}$ incident power on a single unit cell, and the frequency of the pump is eventually fixed close to a resonance frequency $\left(f_{0}=10.6 \mathrm{GHz}\right)$. Figure $\mathrm{S} 8$ in the Supplemental Material [26] shows that the dynamics of the unit cell is insensitive to the specific frequency of the pump. Therefore, only the power of the pump is a relevant parameter that may lead to bifurcations. For a preferred power of operation of $P_{0 \mathrm{op}} \mathrm{W}$ and for deviations of about $\Delta \varphi=1 \mathrm{rad}$, our numerical simulations impose a restoring constant of 


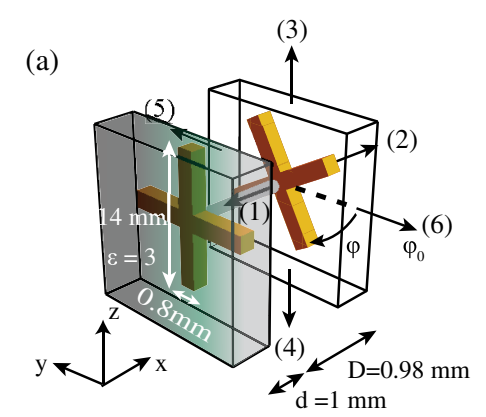

(d)

Phase diagram

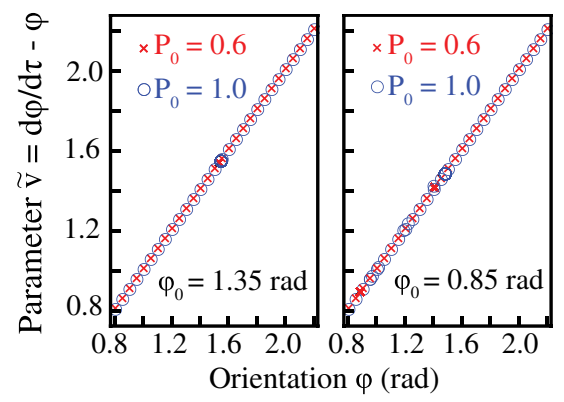

(b) Total torque (J)

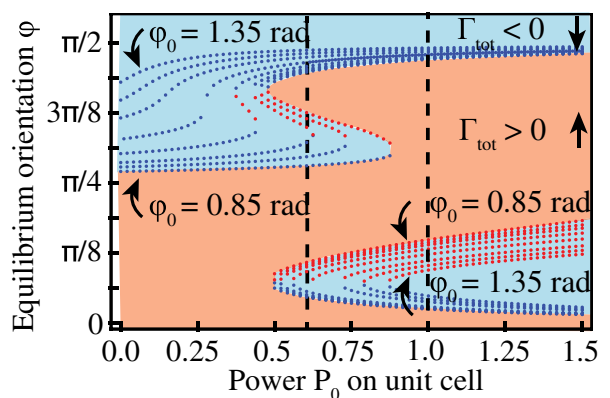

(e) Switching time (s) with $\varphi_{0}=1.35 \mathrm{rad}$

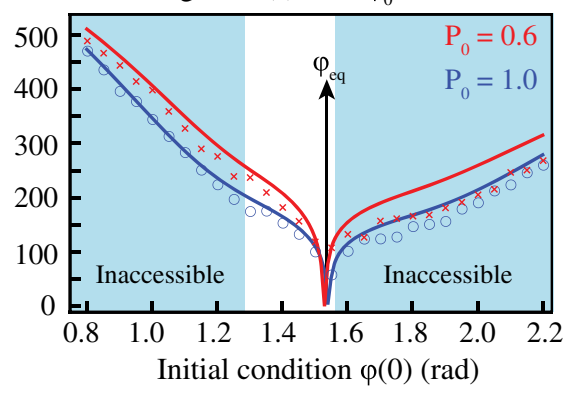

(c) Phase diagram $(\varphi, \mathrm{d} \varphi / \mathrm{d} \tau)$ with $\mathrm{P}_{0}=1.0$

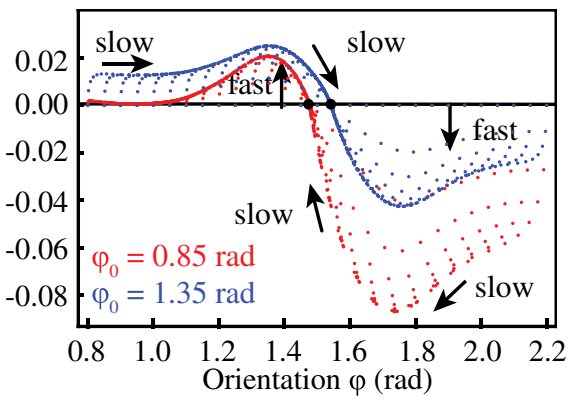

(f) Switching time (s) with $\varphi_{0}=0.85 \mathrm{rad}$

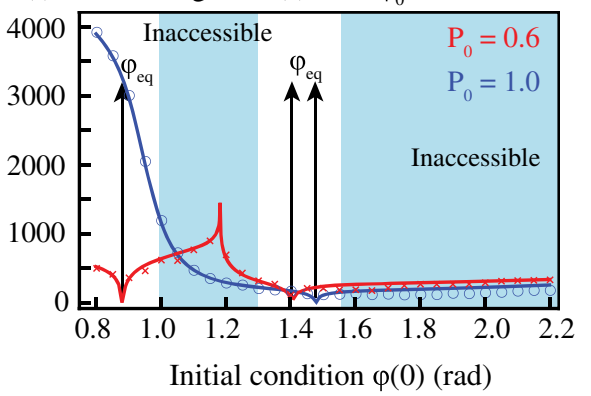

FIG. 2. Transient dynamics of a cross-wire metasurface. (a) An optomechanical cross-wire unit cell with a wire equilibrium $\varphi_{0}$ and an angular orientation $\varphi$ due to pump-induced torques. (b) Nonlinear dependence of stable (blue) and unstable (red) equilibrium states on the pump power $P_{0}$ for several values of $\varphi_{0} \in\{0.85 \mathrm{rad}, \ldots, 1.35 \mathrm{rad}\}$. Blue and orange shaded regions, respectively, indicate when the total torque is positive or negative when $\varphi_{0}=0.85$. (c) Trajectories through phase space for $\varphi_{0}=0.85 \operatorname{rad}$ and $\varphi_{0}=1.35 \operatorname{rad}$ at $P_{0}=1$. Each trajectory starts on the horizontal axis and evolves fast to a fixed curve, along which slow dynamics dominates the reconfiguration time. (d) Visualization of the slow curve in alternative coordinates $(\varphi, \tilde{v})$ leads to a diagonal line. (e), (f) Numerical (circles) and analytic (solid curves) predictions of the configuration time for $\varphi_{0}=1.35 \mathrm{rad}$ and $\varphi_{0}=0.85 \mathrm{rad}$ with $P_{0}=0.6$ (red) and $P_{0}=1$ (blue).

$\kappa=3.6 \times 10^{-4} P_{0 \text { op }}$. In the following, the use of $P_{0 \text { op }}=1$ does not affect the generality of the results. Figure 2(b) confirms that the cross-wire metasurface has a hysteresis curve with stable (blue) and unstable (red) equilibrium orientations for several values of the equilibrium parameter $\varphi_{0}$. Note that the wires experience a (counter) clockwise rotation in the orange (blue) area, leading to an upward (downward) trajectory in the graph. Therefore, bifurcations in the lower part of Fig. 2(b) and unstable states in the upper part of Fig. 2(b) cannot be reached by changing the pump power and will be excluded by the blue regions in Figs. 2(e) and 2(f). Figure 2(b) further shows that when $\varphi_{0}$ is smaller than $1.03 \mathrm{rad}$, there are bifurcation points where solutions are created or merged. Figure 2(c) visualizes a collection of trajectories through phase space for $P_{0}=1$, each with different initial conditions on the horizontal axis. Trajectories in red (blue) correspond to an equilibrium parameter $\varphi_{0}=0.85 \mathrm{rad}\left(\varphi_{0}=1.35 \mathrm{rad}\right)$ of the wire, for which there are (no) bifurcations. In both cases, trajectories quickly converge to a specific curve before moving slowly toward a single equilibrium [Fig. 2(c)]. However, in the case of $\varphi_{0}=0.85 \mathrm{rad}$, the trajectory through phase space approaches a bifurcation point and experiences a critical slowing-down, so its angular velocity is strongly reduced. For underdamped systems, the trajectory in phase space connects to the slow curve close to bifurcations, but otherwise spirals down toward equilibrium [see Fig. S4(c) in the Supplemental Material [26] ]. A semianalytical formula for the reconfiguration time $\Delta t$ is derived based on alternative coordinates $(\varphi, \tilde{v})$,

$$
\frac{d \psi}{d \tau}=\tilde{v}-\psi, \frac{d \tilde{v}}{d \tau}=-\tilde{\kappa}\left(\psi-\psi_{0}\right)+\mu \frac{\langle\tilde{\mathcal{A}}(\psi)\rangle}{\left\langle\tilde{\mathcal{A}}_{\max }(\psi)\right\rangle} .
$$

For convenience, we have normalized the electromagnetic action by introducing a parameter $\mu$. For example, a critically damped unit cell at $P_{0}=1$ results in a value $\mu=0.04$ which is very small. Because of this, trajectories in phase space reduce to the diagonal line $\tilde{v}=\varphi[$ Fig. 2(d)]. As a result, the reconfiguration time $\Delta t$ to switch from $\psi_{\mathrm{eq}}^{(1)}$ to $\psi_{\mathrm{eq}}^{(2)}$ with an accuracy of $\Delta=0.1^{\circ}=0.1(\pi / 180)$ rad along this line is given by

$$
\Delta t=\frac{1}{\tilde{\gamma}} \int_{\psi_{\mathrm{eq}}^{(1)}}^{\psi_{\mathrm{eq}}^{(2)}-\Delta} \frac{d \psi}{\mu \tilde{\mathcal{A}}_{\mathrm{norm}}(\psi)-\tilde{\kappa}\left(\psi-\psi_{0}\right)} .
$$

Figures 2(e) and 2(f) contain the reconfiguration time that is required to reach the equilibrium state for $P_{0}=0.6$ (red) and $P_{0}=1$ (blue) from initial orientations on the horizontal axis. 
Equilibrium orientations are easily found by looking for dips in the curve. The semianalytical predictions of Eq. (3) (solid lines) agree very well with exact numerical solutions of the equation of motion (circles and crosses). For small separations, the elastic response of the wire contributes the most to the reconfiguration time, which increases in a logarithmic fashion [Eq. (3)]. At larger separations, contributions due to the nonlinear response of the metasurface dominate, and switching times increase substantially when the net torque is lowest. In particular, in the presence of bifurcations [Fig. 2(f)], initial conditions $\varphi(0)$ close to the unstable equilibrium for $P_{0}=0.6$ induce singular switching times. Although catastrophic initial conditions cannot be reached by increasing or decreasing the pump [Fig. 2(b)], as indicated by the blue regions in Figs. 2(e) and 2(f), hysteresis curves may yet increase reconfiguration times by an order of magnitude. From an application point of view, one would like to determine the time that is required to change the electromagnetic response by a particular amount $\Delta \delta=\delta^{(2)}-\delta^{(1)}$. In Fig. 3(a), the colored dots represent initial orientations $\varphi^{(1)}$ that impose different changes in the electromagnetic response $\Delta \delta$ as they evolve toward the final equilibrium $\varphi^{(2)}$ at $P_{0}=1$ (green dot). Importantly, these states can be reached for all values of $\varphi_{0}$ in Fig. 2(b), without moving too close to the bifurcation points, so the reconfiguration time is mainly limited by the damped motion of the unit cell. The accumulated change $\Delta \delta=\left|\int_{\psi^{(1)}}^{\psi^{(2)}}(\partial \delta / \partial \psi) d \psi\right|$ can be expressed with respect to the reconfiguration time $\Delta t$ by making use of Eq. (3): $\Delta \delta=$ $\tilde{\gamma}\left|\int_{0}^{\Delta t}(\partial \delta / \partial \psi)\left(P_{0} \tilde{\mathcal{A}}(\psi)-\tilde{\kappa}\left(\psi-\psi_{0}\right)\right) d t\right|$. In addition, because the motion of the unit cell in regions with a low electromagnetic action take up the most time, the average $(1 / \Delta \psi)\left|\int(\partial \delta / \partial \psi)\left(P_{0} \tilde{\mathcal{A}}(\psi)-\tilde{\kappa}\left(\psi-\psi_{0}\right)\right) d \psi\right|$ is larger than the time average $(1 / \Delta t) \mid \int(\partial \delta / \partial \psi)\left(P_{0} \tilde{\mathcal{A}}(\psi)-\right.$ $\left.\tilde{\kappa}\left(\psi-\psi_{0}\right)\right) d t \mid$. As a result, we obtain the following lower bound:

$$
\Delta t \geq \frac{1}{\tilde{\gamma}} \frac{|\Delta \delta|\left(\psi^{(2)}-\psi^{(1)}\right)}{\left.\int \frac{d \delta}{d \psi}(\psi)\left[P_{0} \tilde{\mathcal{A}}_{(} \psi\right)-\tilde{\kappa}\left(\psi-\psi_{0}\right)\right] d \psi \mid} .
$$

As expected, the lower bound increases for weak resonances $((d \delta / d \psi)$ small $)$ and low net torques. Note that the previous derivation is only valid when $(d \delta / d \psi) \tilde{\mathcal{A}}$ has a constant sign; i.e., it is not strictly valid when the trajectory crosses a resonance of the response function. Figure 3(b) shows that the reconfiguration time always exceeds the lower bound (black dots), which, in this case, lies slightly below 5 times the characteristic decay time of a critically damped oscillator (gray dots). Surprisingly, but in agreement with Eq. (3), the reconfiguration time improves in the presence of bifurcations, i.e., when metasurface designs implement low values for the restoring parameter $\varphi_{0}$. This is because hysteresis curves push equilibrium states to higher powers [Fig. 2(b)]; (a) Optical rotation (deg)

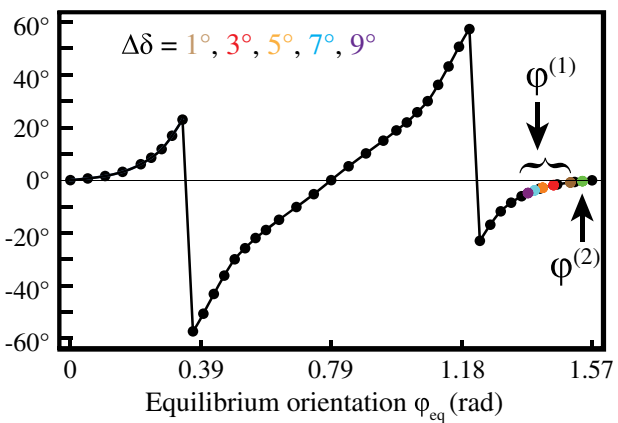

(b) Switching time $\Delta \mathrm{t}(\mathrm{s})$

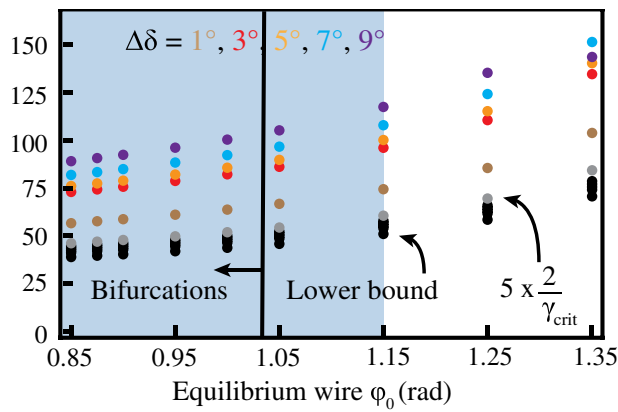

FIG. 3. Relation between a specific change in response $\Delta \delta$ and the reconfiguration time $\Delta t$. (a) Numerical values (black dots) and cubic interpolation (line) of the optical rotation of a signal beam $\delta\left(\varphi_{\mathrm{eq}}\right)$ at equilibrium orientations $\varphi_{\mathrm{eq}}$. Different values $\varphi^{(1)}$ (colored points) correspond to different changes in optical activity $\Delta \delta \in\left\{2^{\circ}, 4^{\circ}, 6^{\circ}, 8^{\circ}, 10^{\circ}\right\}$ as the unit cell evolves to $\varphi^{(2)}$ (green dot). (b) Reconfiguration times of trajectories from $\varphi^{(1)}$ to $\varphi^{(2)}$ for several values of $\varphi_{0}$ and $(\kappa / \mathcal{M})=0.00036$. The lower bound on the reconfiguration time [Eq. (4)] is shown by black dots, and the characteristic time scale for critical damping is shown by gray dots. The blue region indicates that transitions require a pump power which is larger than $2 P_{0 \text { op }}$.

i.e., the power of the pump that is required to reach $\varphi^{(2)}$ is higher for $\varphi_{0}=0.85 \mathrm{rad}$ than for $\varphi_{0}=1.35 \mathrm{rad}$. However, from an experimental point of view, the power of operation may be limited. For example, the blue region contains those parameter values $\varphi_{0}<1.16$ for which the power of the pump is at least twice the desired power of operation $P_{0 \text { op. }}$. If moderate changes in power are required, bifurcations should be avoided.

In the Supplemental Material [26], we derive scaling laws for the reconfiguration time in terms of the resonance frequency $f$ and the intensity of the pump $I$ : $\Delta t=\left[\left(f / f_{0}\right)\right]^{\alpha}\left[\left(I / I_{0}\right)\right]^{-1 / 2} \Delta t_{0}$, with $\alpha=-1 / 2(-1)$ for torque-based (force-based) optomechanical unit cells. For example, an optical cross-wire metasurface with parameters based on Ref. [10], i.e., $f=2.0 \times 10^{14} \mathrm{~Hz}$ and $I=31 \mathrm{~W} / \mathrm{mm}^{2}$ converges at best in $4.4 \mathrm{~ms}$ when using $\Delta t_{0}=50 \mathrm{~s}$ from Fig. $3(\mathrm{~b}), f_{0}=10.6 \mathrm{GHz}$, and $I_{0}=(1 \mathrm{~W} / 225) \mathrm{mm}^{2}$. Force-based optomechanical metasurfaces have a slightly better scaling that would lead to $\Delta t=0.63 \times 10^{-6} \Delta t_{0}$. This roughly corresponds 
to a maximum bandwidth of a few $\mathrm{MHz}$, in agreement with Ref. [10] but still 3 orders of magnitude lower than state-ofthe-art silicon switches [20].

In conclusion, the transient dynamics of optomechanical metasurfaces is adversely affected both by the presence of bifurcations (Fig. 2) and by the slow exponential convergence of damped mechanical elements [Eq. (3)]. In particular, critical slowing-down may restrict the reconfiguration time by over an order of magnitude. In addition, the lower bound for the reconfiguration time [Eq. (4) and Fig. 3] scales slowly with the resonance frequency and the intensity on the unit cell, so optomechanical metasurfaces cannot be used as fast switches, even at optical frequencies. To boost switching times, one may (1) try to manipulate particles separately with highly confined fields [2], (2) use other mechanisms for tuning metasurface properties $[21,42]$, or (3) generate dynamic optical responses through a continuous modulation of the pump beam [10,11]. However, also for modulated systems, nonlinear dynamics may restrict the speed by which one may switch from one modulation pattern to another.

S. V. and V.G. acknowledge fellowships from the Research Foundation Flanders (FWO-Vlaanderen). Work at VUB was partially supported by the Research Council of the VUB and by the Interuniversity Attraction Poles programme of the Belgian Science Policy Office, under Grant No. IAP P7-35. Work at Chalmers was partially supported by Vetenskapsrådet under Grant No. 2016-03603.

ginis@ seas.harvard.edu

[1] T. J. Kippenberg and K. J. Vahala, Science 321, 1172 (2008).

[2] M. Aspelmeyer, T. J. Kippenberg, and F. Marquardt, Rev. Mod. Phys. 86, 1391 (2014).

[3] A. Ashkin, Phys. Rev. Lett. 40, 729 (1978).

[4] B. P. Abbott, R. Abbott, T. D. Abbott, M. R. Abernathy, F. Acernese, K. Ackley, C. Adams, T. Adams, P. Addesso et al. (LIGO Scientific Collaboration), Phys. Rev. Lett. 116, 221101 (2016).

[5] L. Liu, S. Kheifets, V. Ginis, and F. Capasso, Phys. Rev. Lett. 116, 228001 (2016).

[6] M. Lapine, I. V. Shadrivov, D. A. Powell, and Y. S. Kivshar, Nat. Mater. 11, 30 (2012).

[7] R. Zhao, P. Tassin, T. Koschny, and C. M. Soukoulis, Opt. Express 18, 25665 (2010).

[8] N. Liu, H. Liu, S. Zhu, and H. Giessen, Nat. Photonics 3, 157 (2009).

[9] M. Liu, Y. Sun, D. A. Powell, I. V. Shadrivov, M. Lapine, R. C. McPhedran, and Y.S. Kivshar, Phys. Rev. B 87, 235126 (2013).

[10] A. Karvounis, J. Ou, W. Wu, K. F. MacDonald, and N. I. Zheludev, Appl. Phys. Lett. 107, 191110 (2015).

[11] J. Ou, E. Plum, J. Zhang, and N. I. Zheludev, Adv. Mater. 28, 729 (2016).
[12] M. Liu, D. A. Powell, R. Guo, I. V. Shadrivov, and Y. S. Kivshar, Adv. Opt. Mater. 5, 1600760 (2017).

[13] M. Lapine, I. V. Shadrivov, D. A. Powell, and Y. S. Kivshar, Sci. Rep. 1, 138 (2011).

[14] A. P. Slobozhanyuk, M. Lapine, D. A. Powell, I. V. Shadrivov, Y. S. Kivshar, R. C. McPhedran, and P. A. Belov, Adv. Mater. 25, 3409 (2013).

[15] M. Liu, D. A. Powell, I. V. Shadrivov, M. Lapine, and Y. S. Kivshar, Nat. Commun. 5, 4441 (2014).

[16] A. Belardini, M. Centini, G. Leahu, D. C. Hooper, R. L. Voti, E. Fazio, J. W. Haus, A. Sarangan, V. K. Valev, and C. Sibilia, Sci. Rep. 6, 31796 (2016).

[17] D. A. B. Miller, Nat. Photonics 4, 3 (2010).

[18] W. Chen, K. M. Beck, R. Bücker, M. Gullans, M. D. Lukin, H. Tanji-Suzuki, and V. Vuletić, Science 341, 768 (2013).

[19] L. H. Nicholls, F. J. Rodríguez-Fortuño, M. E. Nasir, R. M. Córdova-Castro, N. Olivier, G. A. Wurtz, and A. V. Zayats, Nat. Photonics 11, 628 (2017).

[20] G. T. Reed, G. Mashanovich, F. Y. Gardes, and D. J. Thomson, Nat. Photonics 4, 518 (2010).

[21] M. Lapine, I. V. Shadrivov, and Y. S. Kivshar, Rev. Mod. Phys. 86, 1093 (2014).

[22] A. H. Nayfeh and D. T. Mook, Nonlinear Oscillations (John Wiley \& Sons, New York, 1995).

[23] S. H. Strogatz, Nonlinear Dynamics and Chaos: With Applications to Physics, Biology, Chemistry, and Engineering (Westview Press, Cambridge, 2014).

[24] M. C. Soriano, J. García-Ojalvo, C. R. Mirasso, and I. Fischer, Rev. Mod. Phys. 85, 421 (2013).

[25] I. Novikova, Physics 2, 7 (2009).

[26] See Supplemental Material at http://link.aps.org/ supplemental/10.1103/PhysRevLett.120.197402 providing technical information about finite-element simulations and the nonlinear dynamics of the cross-wire metasurface.

[27] I. S. N. Murty, Int. J. Nonlinear Mech. 6, 45 (1971).

[28] Z. Wang, F. Cheng, T. Winsor, and Y. Liu, Nat. Nanotechnol. 27, 412001 (2016).

[29] J. Zhou, J. Dong, B. Wang, T. Koschny, M. Kafesaki, and C.M. Soukoulis, Phys. Rev. B 79, 121104 (2009).

[30] H. Chen, A. J. Taylor, and N. Yu, Rep. Prog. Phys. 79, 076401 (2016).

[31] H. Chen, W. J. Padilla, J. M. O. Zide, A. C. Gossard, A. J. Taylor, and R. D. Averitt, Nature (London) 444, 597 (2006).

[32] J. Zhou, D. R. Chowdhury, R. Zhao, A. K. Azad, H.-T. Chen, C. M. Soukoulis, A. J. Taylor, and J. F. O'Hara, Phys. Rev. B 86, 035448 (2012).

[33] S. Zhang, J. Zhou, Y. Park, J. Rho, R. Singh, S. Nam, A. K. Azad, H. Chen, X. Yin, and A. J. Taylor, Nat. Commun. 3, 942 (2012).

[34] S. A. Mousavi, E. Plum, J. Shi, and N. I. Zheludev, Appl. Phys. Lett. 105, 011906 (2014).

[35] A. Kuzyk, R. Schreiber, H. Zhang, A. O. Govorov, T. Liedl, and N. Liu, Nat. Mater. 13, 862 (2014).

[36] X. Yin, M. Schaferling, A. U. Michel, A. Tittl, M. Wuttig, T. Taubner, and H. Giessen, Nano Lett. 15, 4255 (2015). 
[37] A. Komar, Z. Fang, J. Bohn, J. Sautter, M. Decker, A. Miroshnichenko, T. Pertsch, I. Brener, Y. S. Kivshar, I. Staude et al., Appl. Phys. Lett. 110, 071109 (2017).

[38] A. Lakhtakia and W. S. Weiglhofer, Introduction to Complex Mediums for Optics and Electromagnetics (SPIE Optical Engineering Press, Bellingham, Washington, 2003), Vol. 123.

[39] P. Tassin, T. Koschny, and C. M. Soukoulis, Physica (Amsterdam) 407B, 4062 (2012).
[40] W. H. Press, S. A. Teukolsky, W. T. Vetterling, and B. P. Flannery, Numerical Recipes in $C$ (Cambridge University Press Cambridge, England, 1996), Vol. 2.

[41] M. A. Ordal, L. L. Long, R. J. Bell, S. E. Bell, R. R. Bell, R. W. Alexander, and C. A. Ward, Appl. Opt. 22, 1099 (1983).

[42] N. I. Zheludev and E. Plum, Nat. Nanotechnol. 11, 16 (2016). 